It is a 'pull' in the first case and a 'push' in the second.

The process in III is assisted by the known lability of $\mathrm{OH}$ in the system $\mathrm{N}-\mathrm{C}-\mathrm{OH}$.

The rearrangement of I to II is evidently parallel to that of III to IV and it is tempting to assume hydrolysis of the epoxide to a glycol so as to achieve a precise analogy. However, the reaction may be more direct and dependent on the loss of one of the available protons (on NH, or $\mathrm{OH}$ ) and on the stability of the anion so produced. The charge may then be transferred to the epoxide oxygen, which is enabled to break away from $C_{2}$ and take the covalency electrons with it, the rearrangement following automatically.

We take this opportunity of making a preliminary announcement of the synthesis of cinchonamine (formula of Prelog et al. ${ }^{2}$ ) via quinamicine by coupling of tryptophol with meroquinene. Quinamicine is crude quinamidine, and we have not yet confirmed one link in the synthetic chain, namely, the recorded transformation of quinamicine into apoquinamine.

Note added in proof. Quite recently Witkop has modified the quinamine formula so as to include a furanoid ring and hydroxyl in position-3. This is acceptable and in the above 'epoxide' should be 'cyclic ether'.

C. C. J. Culvenor

L. J. GOLDSWORTHY

K. S. KIRBY

R. ROBINSON

Dyson Perrins Laboratory,

University of Oxford. March 13.

1 Kirby, J. Chem. Soc., 735 (1949).

- Kirby, J. Chem. Soc., 528 (1945). Goutarel, Janot, Prelog and Taylor, Helv. Chim. Acta, 33, 150 (1950). Bendz, Miss G., Culvenor,
Goldsworthy, Kirby and Robinson; Paul Karrer Festschrift, Goldsworthy, Kirby and Robinson; Paul Karrer
Zurich (1949); J. Chem. Soc. (in the press, 1950).

Plant and Robinson, Nature, 165, 36 (1950). cf. Witkop, J. Amer. Chem. Soc., $72,614(1950)$.

\section{Synthesis of Ergothioneine}

ERGOTHIONEINE has been isolated from ergot ${ }^{1}$ and from mammalian blood ${ }^{2,3}$. Barger and Ewins ${ }^{4}$ and Akabori ${ }^{5}$ showed that the structure of ergothioneine (I) is $\beta$-2-thiolglyoxaline-4(5)-propiobetaine. This structure has now been confirmed by synthesis according to the following scheme.

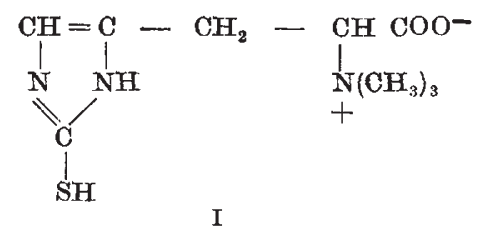

2-Thiol histidine was prepared by Ashley and Harington's ${ }^{6}$ method with modifications which improved the yield (120 gm. histidine monohydrochloride monohydrate yielded $21 \cdot 0 \mathrm{gm}$. 2-thiol histidine). By treating with chlorformic ester in absolute alcohol, this was converted to 2-S-carbethoxy histidine dihydrochloride (II) (m.p. $189^{\circ}$, dec.), in which the sulphur atom was protected by the easily removable carbethoxy group.

Methylation of (II) with silver oxide and methyl iodide gave the silver complex of 2 -S-carbethoxy histidine betaine.

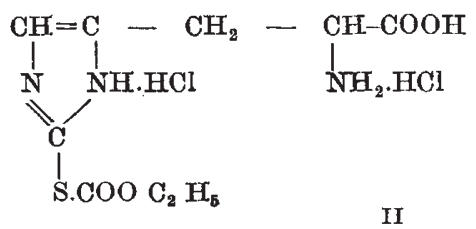

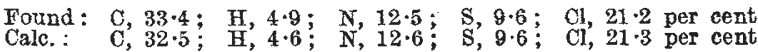

Boiling with $3 N$ hydrochloric acid brought about hydrolysis and decarboxylation. The solution was freed from silver with hydrogen sulphide and the ergothioneine (I) isolated as a phosphotungstate, by precipitation with 10 per cent phosphotungstic acid in 5 per cent sulphuric acid. This was decomposed with baryta, excess baryta being removed with an equivalent amount of sulphuric acid. The ergothioneine thus obtained was twice recrystallized from aqueous alcohol (m.p. $290^{\circ}$ decomp.).

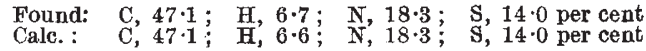

In the following tests, synthetic ergothioneine and specimens of ergothioneine from blood (Hunter) and ergot (Lawson) showed identical behaviour : Hunter's ${ }^{7}$ diazo test, sulphur dioxide in acid solution, gold chloride, iodine in potassium iodide solution, mercuric chloride solution, and Mayer's alkaloid reagent. On warming with $2 \mathrm{~N}$ sodium hydroxide, trimethylamine was evolved leaving a yellow solution. Addition of Folin-Marenzi's uric acid reagent to a solution in sodium carbonate produced an immediate blue colour.

No differences in $R_{F}$ values were detected when natural and synthetic ergothioneine were subjected to paper partition chromatography ${ }^{8}$ in collidine or in phenol.

The ultra-violet absorption spectre in water were identical, with a maximum at $258 \mathrm{~m} \mu, E_{\mathrm{mol} .}^{1 \mathrm{~cm} .}=1 \cdot 6 \times$ 104. A full report of this work will be published elsewhere.

We wish to express our gratitude to Mr. P. R. E. Wallace for technical assistance throughout the synthesis, to Dr. E. Work and Mr. F. Grover for the paper chromatograms, to Dr. G. Hunter for help in isolation in the final stages, and to Drs. Weiler and Strauss of Oxford for the microanalyses. This work was performed during the tenure by one of us (H. H.) of a British Drug Houses Research Fellowship.

\section{H. Heath \\ A. LAWSON \\ C. Rimington}

Department of Chemical Pathology,

University College Hospital Medical School, London, W.C.1, and

Department of Chemistry,

Royal Free Hospital School of Medicine, London, W.C.1. May 26.

${ }^{1}$ Tanret, G., J. Pharm. Chim., 30, 145 (1909).

"Hunter, G., and Eagles, B. A.. J. Biol. Chem., 72, 123 (1927).

s Newton, E. B.. Benedict, S. R., and Dakin, H. D., J. Biol. Chem., 72, 367 (1927).

- Barger, G., and Ewins, A. J., J. Chem. Soc., 99, 2336 (1911).

${ }^{5}$ Akabori, S., Ber. deut. chem. Ge8., 66, 151 (1933).

- Ashley, J. N., and Harington, C. R., J. Chem. Soc., 2586 (1930).

7 Hunter, G., Biochem. J., 22, 4 (1928).

"Work, E., Lancet, (i), 652 (1949). 